\title{
Negative-sequence Eddy Current Field Calculation for the World's First AP1000 Nuclear Half-speed Generator
}

\author{
Bao-jun $\mathrm{Ge}^{1}, \mathrm{Wu}$ Guo $^{1 *}$, Jian Guo ${ }^{2}$ and Dan-hui Zhang ${ }^{1}$ \\ ${ }^{1}$ Collage of Electrical and Electronic Engineering \\ Harbin University of Science and Technology, Harbin, China, 150080 \\ ${ }^{2}$ Collage of Architecture and Civil Engineering \\ Qiqihar University, Qiqihar, China, 161006 \\ guowuhaligong@126.com
}

\begin{abstract}
The negative-sequence eddy current field of the first AP1000 third-generation 1,250 MVA nuclear half-speed (4-pole) turbo-generator is deeply researched in this paper. In order to find out the influence rules of using different rotor slot wedge materials, the eddy current loss densities, which are generated by negative-sequence current, are compared and analyzed. It is well known that aluminium and aluminum-bronze are widespread materials in electric machine industry. Hence, the two materials are adopted respectively as the slot wedge material, the eddy current loss calculations of slot wedges, rotor teeth and damping bars are given, and the loss characteristic analysis results are also obtained in this work. The calculation and analysis results indicate that different materials have different effects on eddy current loss, especially for the loss density of slot wedges, eddy current loss density of the big tooth area is bigger than the small tooth, and damping bars have the biggest loss density on the rotor surface, which all should be attached importance to in actual large capacity generator design.
\end{abstract}

Keywords: negative-sequence, eddy current field, wedge materials, aluminium, aluminum-bronze, AP1000 generator

\section{Introduction}

With the development of motor manufacturing's production technology, the capacity of large generators are increasing as well, the generator efficiency can reach more than $98 \%$, and all kinds of losses which also increase correspondingly are inevitable. The increasing capacity leads to the structure more complex, and the unbalance operation causes even more serious damages on the generator $[1,2]$. Hence, the destruction of the eddy current loss, which produced by negative sequence current in the generator rotor surface, cannot be ignored. However, the large capacity motor is expensive, so how to avoid the harm of asymmetric operation for the generator rotor is particularly important in electric machine industry.

Generally, turbine generators play an important role in network operation, but for a variety of reasons, generators carry three-phase unbalanced load sometimes. Generator stator windings will produce negative sequence current in asymmetric operation. Since the rotary magnetic field generated by the negative sequence current is rotating at the same speed, inverting with the rotor, thus, the negative sequence field will induce a series of harmonics in the rotor winding [3]. With the motor industry development, this situation has become increasingly prominent and urgent.

Due to the corresponding negative sequence component induced by each harmonic, stator and rotor will all contain multiple harmonics owing to this interaction. Meanwhile, because the negative sequence magnetic field speed is twice the synchronous speed frequency, eddy current on the rotor surface is induced, 
which can cause rotor heating and even damage generator system [4-8]. Hence, some issues of eddy current field for half-speed large turbine generators are analyzed and researched very closely in this paper.

\section{AP1000 Generator Analysis}

\subsection{Eddy Current Field Model of AP1000 Generator}

The typical half-speed large generator, AP1000 nuclear generator, is adopted as an example in this paper, to carry out the analysis and calculation of eddy current loss density.

Half-speed turbine generator electromagnetic distribution is very complex. Therefore, in order to simplify calculation, the two dimensional finite element analysis of electromagnetic field is used, and the assumptions are as follow:

(1). the rotor axial length is much longer than the penetration depth induced current in the rotor surface. It can be assumed that the motor is infinite, and only considered the axial (Direction Z) rotor current distribution in the two-dimensional field analysis.

(2). Stator core permeability $\mu=\infty$, conductivity $\sigma=0$, the conductivity of the rotor core $\sigma=$ const , and the nonlinear effect is ignored.

(3). the eddy current of stator vortex laminated core and source current region is negligible.

The entire cross section of generator is taken for electromagnetic field calculation, which is shown in Figure 1.

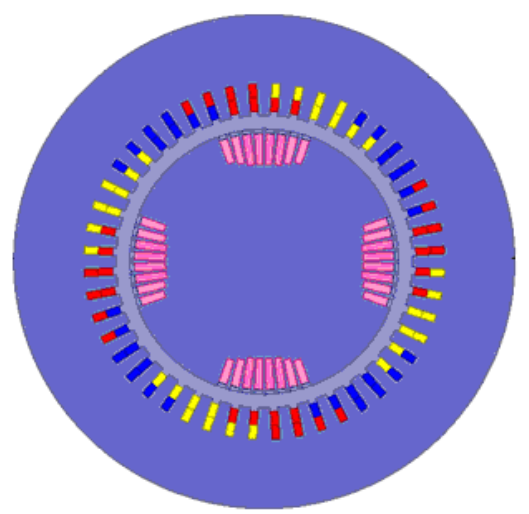

Figure 1. 2D Physical Model of Solved Region

In order to satisfy the boundary value problem, the finite element method is used to solve the electromagnetic parameters together with the corresponding boundary conditions as follow:

$\left\{\begin{array}{l}\Omega: \frac{\partial}{\partial x}\left(\frac{1}{\mu} \frac{\partial A_{z}}{\partial x}\right)+\frac{\partial}{\partial y}\left(\frac{1}{\mu} \frac{\partial A_{z}}{\partial y}\right)=-J_{\mathrm{s}}+j \omega \omega A_{\mathrm{z}} \\ s_{1}:\left.A_{z}\right|_{\Gamma_{1}}=0\end{array}\right.$

Where:

$J_{\mathrm{s}}$ is the source current density;

$\mu$ is permeability;

$A_{\mathrm{z}}$ is magnetic vector's direction $\mathrm{Z}$ component;

$\Gamma_{1}$ is the first boundary condition. 
Rotor winding heating will not increase in the running process, which has little effect on the normal operation. Therefore, the eddy loss density calculations, in this paper, only contain the slot wedge, rotor teeth and damping winding part.

\subsection{Negative-Sequence Magnetic Field Characteristics}

The AP1000 generator's system is asymmetry in the unbalance running situation. In this time, this asymmetry system can be decomposed into three symmetric independent networks, which are positive-sequence, negative-sequence and zero-sequence network. The positive-sequence current rotating magnetic field has the same rotating direction with generator rotor, their speeds are also the same, and there is no relative motion. Generally, the main transformer of the generator side has the triangle connection, therefore the influence of zero-sequence component on the generator cannot be considered in the actual operation.

In the air gap between the stator and the rotor of the generator, a rotating magnetic field generated by the negative-sequence current, has the different rotating direction with the rotor synchronous speed, when the rotor is still at the positive synchronous speed. Hence, the rotating magnetic field generated by the negative-sequence current is two times of synchronous speed relative to the rotor, and the 2 times frequency negative-sequence current is inducted on the rotor surface. The induced current is with high frequency, its skin effect is obvious, and the penetrated depth into the rotor surface is shallow [9-11]. Therefore, the current can only flow in the thin layer of the rotor surface, and the penetration depth can be expressed as follow:

$$
h=\sqrt{\frac{2 \rho}{\omega \mu}}
$$

Where:

$\rho$ is the resistance coefficient of material, $\Omega \cdot \mathrm{m}$;

$\omega$ is the angular frequency of the rotor eddy current, $\mathrm{rad} / \mathrm{s}$;

$\mu$ stants $\mu=\mu^{\prime} \mu_{0}$, Magnetic permeability, $\mathrm{h} / \mathrm{m}, \mu_{0}=4 \pi \times 10^{-7} \mathrm{~h} / \mathrm{m}$;

$\mu^{\prime}$ is the relative magnetic conduction coefficient of material.

The negative-sequence current is got when the load current is imbalance, and then the eddy current with 2 times frequency is induced on the rotor surface. Finally, the eddy current loss is produced on the rotor surface and the rotor body's temperature increases. Hence, the accurate calculation of eddy current loss generated by negative-sequence current in the rotor body is particularly important.

In order to reflect the eddy current loss produced by stator negative-sequence current in the rotor surface more accurately, the rotor body surface is divided into different regions considering each part penetration depth of the rotor body surface. Hence, different positions' eddy current losses of the rotor body caused by the negative-sequence current are calculated respectively, such as slot wedges, big teeth, small teeth and damping bars.

Because the rotor body and the wedge use different materials, the penetration depth of slot wedges is larger than the penetration depth of rotor body according to Formula (2). In order to clearly descript each part of the rotor body, big teeth are numbered as $t d_{\mathrm{i}}(i=1,2,3,4)$, small teeth are numbered as $t x_{\mathrm{j}}(j=1,2, \ldots, 28)$, and damping bars are numbered as $d_{\mathrm{m}}(m=1,2, \ldots, 32)$. The slot wedge area is large and its material is different from the rotor body. Hence, aim at accurately calculating the slot wedge loss density of each position, the slot wedge is divided into top, middle and bottom parts, 
which are numbered as $w s_{\mathrm{k}}, w z_{\mathrm{k}}$ and $w x_{\mathrm{k}}(k=1,2, \ldots, 32)$. As shown in Figure 2, the position is No. 1 and along the clockwise direction the number is increasing.

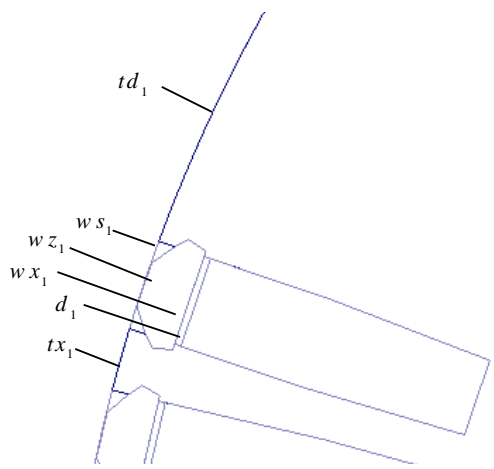

Figure 2. The Subdivision of Rotor

From design data of AP1000 generator [3], it can be seen clearly that the negativesequence capability of this generator is not more than $8 \%$. Hence, $8 \%$ is taken as the negative-sequence capability of AP1000 generator to apply in the research, and the negative-sequence current in the generator stator winding is $8 \%$ of the rated current, that is $I_{2}=0.8 I_{N}$.

The rotation direction of the rotor is opposite to the negative-sequence field rotation direction. By using the equivalent method to deal with the relative motion processing among the two rotations, the rotor is equivalent to a static state, and the negative-sequence magnetic field is in 2 times frequency of the synchronous speed compared with the relatively static rotor [12-16]. Hence, the finite element analysis method is adopted to calculate the rotor eddy current loss, the rotor is equivalent to the static simulation, and the negative-sequence field is in the generator stator winding is electrified with the 2 times frequency current.

\section{Eddy Current Losses Caused By Negative-Sequence Current}

In the asymmetric condition, the rotor excitation winding has less eddy current loss than other parts of the generator rotor, whose value is only the stator negative sequence current's $2 \% \sim 4 \%$. And its heating is not increasing in the operation process, and has little effect on the normal operation. Therefore, the loss density for rotor slot wedge, big and small teeth and damping winding are calculated in this section.

\subsection{Eddy Current Loss Calculation of Slot Wedges}

From the loss density results, as shown in Figure 3, by using the finite element method, the loss density values of each unit in the rotor are different.

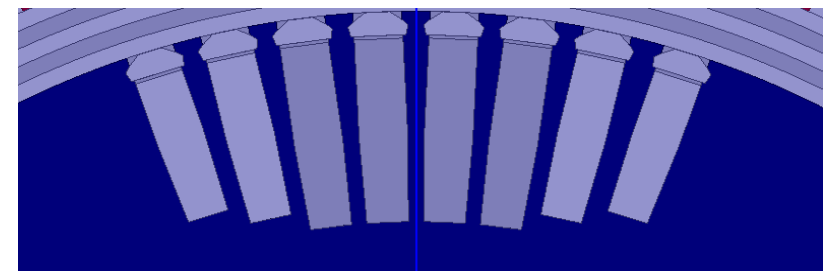

a. Slot Wedges of one Pole

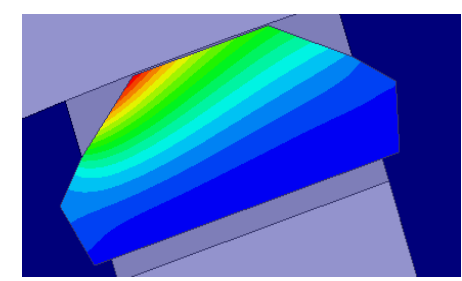

b. Wedge of the Left Part 


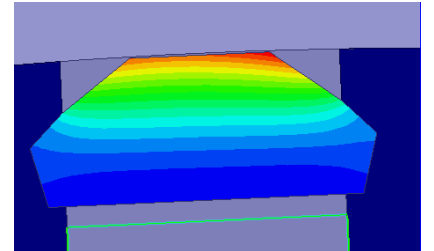

c. Wedge of the Middle Part

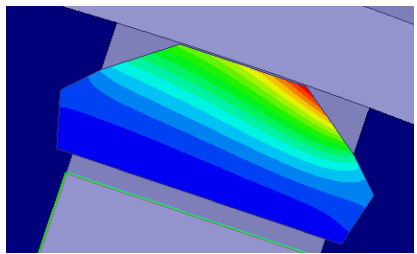

d. Wedge of the Right Part

Figure 3. The Loss Density Distribution of One Pole's Wedges

To bring a convenience and have the data done merely expediently, the same loss density value is given to deal with any part of the same rotor surface. Then, the loss density distributions of wedge's each part are got in the case of using aluminum and aluminum-bronze as the wedge material respectively. The results are shown in Figure 4.

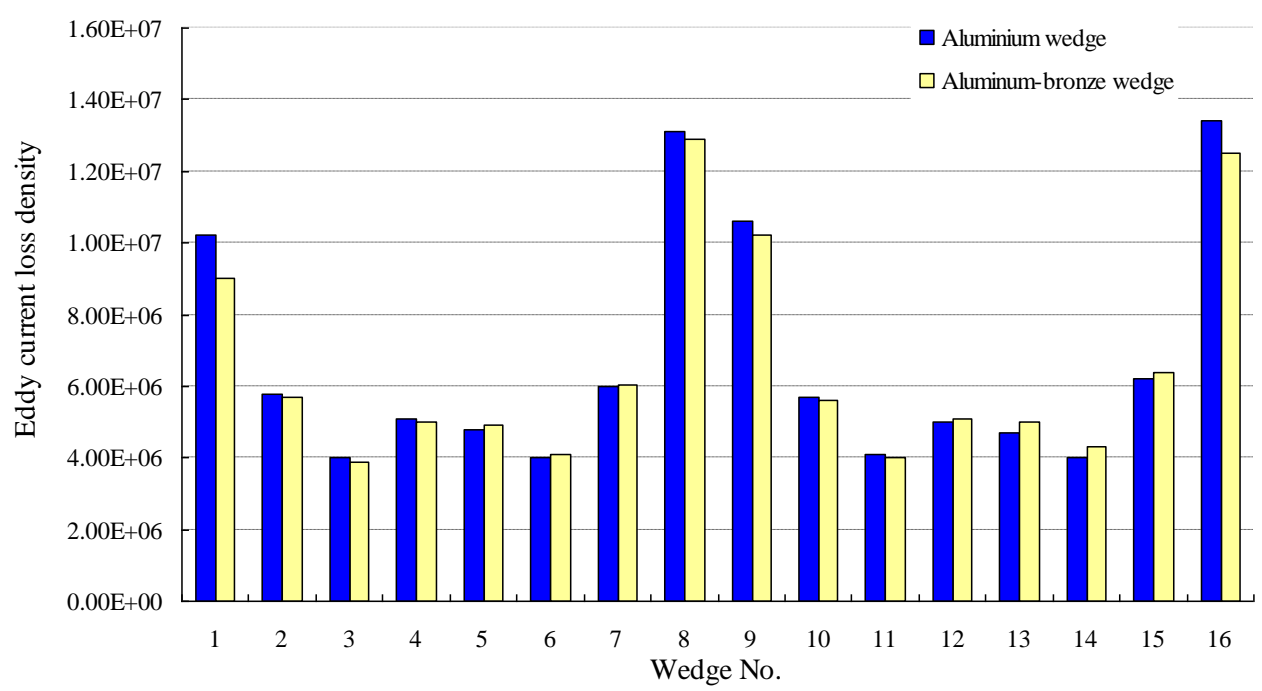

A. The Loss Density of the Top Part

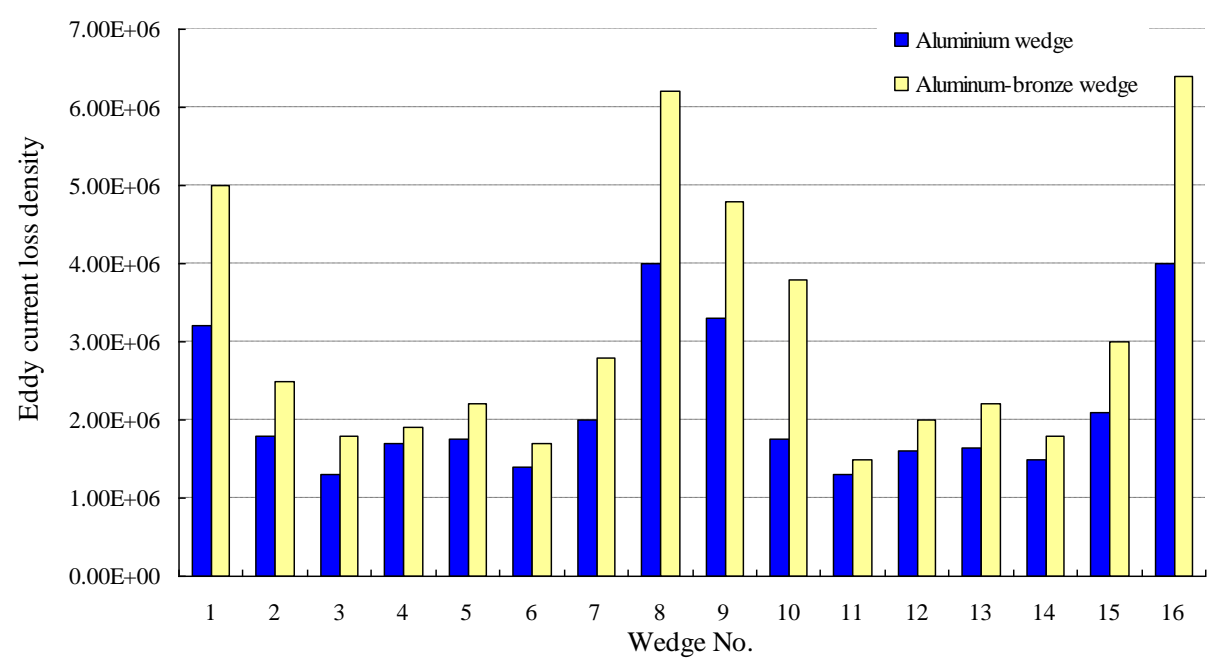

B. The Loss Density of the Middle Part 


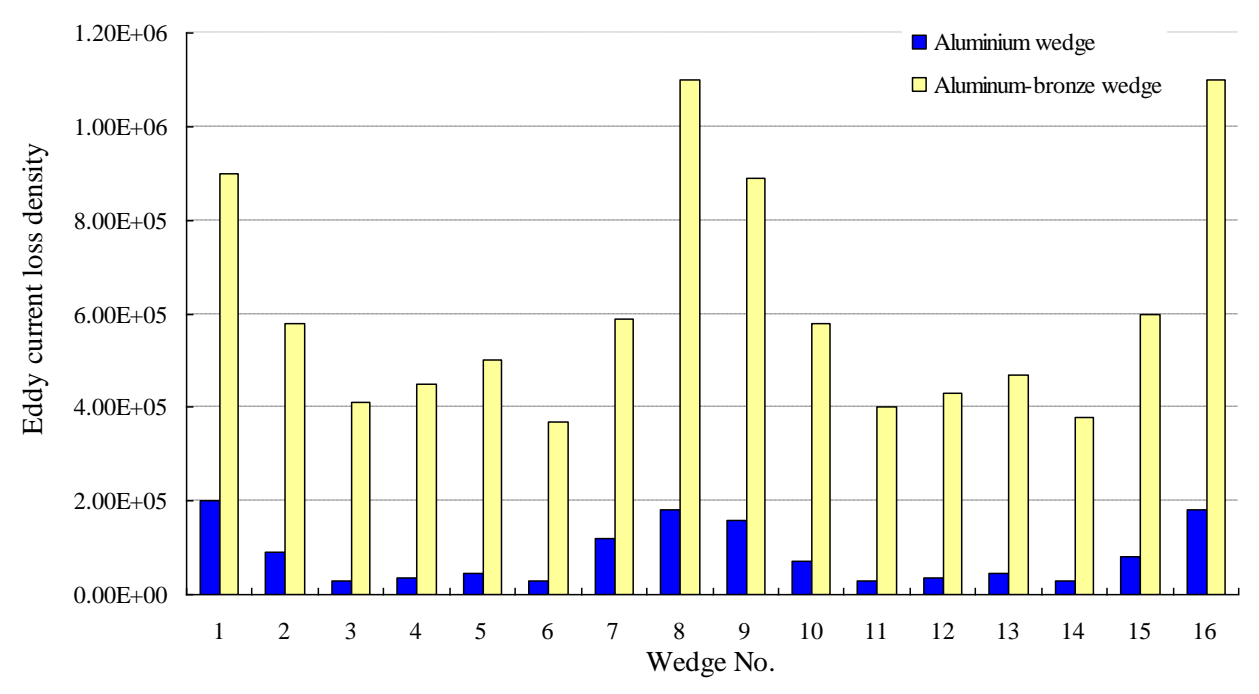

C. The Loss Density of the Bottom

Figure 4. The Loss Density Distribution of Each Portion of the Wedge

From Figure 3 and 4 above, it can be seen clearly that, with the increase of depth from rotor surface, the eddy current generated by negative sequence magnetic field in each part of the rotor is decreased, and the loss of corresponding parts are also reduced. The eddy current values of wedge No.1, No.8, No.9 and No.16, which are near the big teeth, are much higher than other wedges. Furthermore, the eddy current loss density is reduced at different degree of the same depth according to different materials.

First of all, as Figure 3 and 4 shown, the eddy current loss density of wedge's each part has its own variation law. The results indicate that the top part has the maximum value, and the loss density decreases with depth. The reason is the depth from each part of the slot wedge to the rotor surface increases and the strength of the magnetic field generated by negative-sequence current gets smaller as well. When the distance from the rotor surface reaches a certain depth, the negative-sequence field becomes very weak. Therefore, with the increase distance from the rotor surface, the negative-sequence magnetic field generated in each part of the rotor is decreased, and the losses of corresponding parts are reduced gradually.

Second, the eddy current loss density values of slot wedge No.1, 8, 9 and 16 are much higher than any other slot wedges, and these slot wedges are all near the big tooth. In the actual operation of AP1000 generator, the current density of the slot wedge near the big tooth, inducted by negative sequence field is far greater than the other slot wedges in other locations. It is obviously that the loss density values of the slot wedges near the big tooth must be higher than the loss densities in the other positions of the rotor surface.

Third, the loss density value is not only related to the slot wedge position, but also more closely related to the slot wedge material. As can be seen from Figure 4 a), the two loss densities of the upper part by using different slot wedge materials, aluminium and aluminum-bronze respectively, do not have much differences in the value. In that situation, the slot wedge material has less impact on the eddy current loss density value of the slot wedge's top part. Meanwhile, from the results obtained of Figure $4 \mathrm{~b}$ ) and c), the loss density changes of the slot wedges' middle and bottom parts are more significant in the two cases, especially for the bottom part's loss density of the slot wedges, and the loss density of the aluminum-bronze wedge is significantly greater than the aluminum wedge. Using different slot wedge materials leads to the change of slot wedge resistivity, and the resistivity has direct effect on the penetration depth of the eddy current as shown in Formula (2). 
Furthermore, in the same depth, the eddy currents are not same in different materials of the slot wedges, and the negative-sequence fields reduce at different degrees. Hence, the eddy current loss densities of the slot wedge's same part differ from each other in using different materials.

\subsection{Eddy Current Loss Calculation of Rotor Teeth}

Considering the half-speed nuclear generator symmetry, the eddy current loss density distributions of one pair poles' big and small teeth are given in Figure 5 and 6.



Figure 5. The Loss Density Distribution of Small Teeth

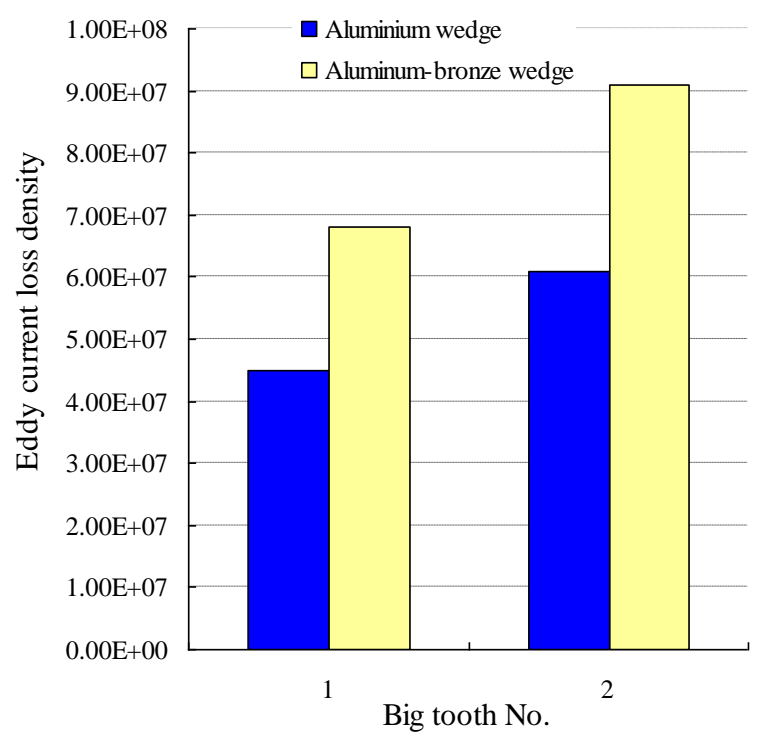

Figure 6. The Loss Density Distribution of Big Teeth

From Figure 5 and 6, it can be concluded that, the eddy current losses of big and small teeth in the situation of using aluminum-bronze slot wedges wedge are bigger than using aluminum slot wedges. Meanwhile, the eddy current loss density of big teeth in the core is larger than the small teeth's value, because the whole area of big 
teeth is larger, and the eddy current loss density in the larger area is bigger than the small teeth area. The per unit area eddy current of big teeth is also bigger than the small teeth. Hence, as the Figure 5 and 6's results shown, the eddy current loss density of big teeth is greater than the eddy current loss of the small teeth.

\subsection{Eddy Current Loss Calculation of Damping Bars}

Additionally, the eddy current losses of damping bars are obtained, which are shown in Figure 7 and 8 , by using the same method.

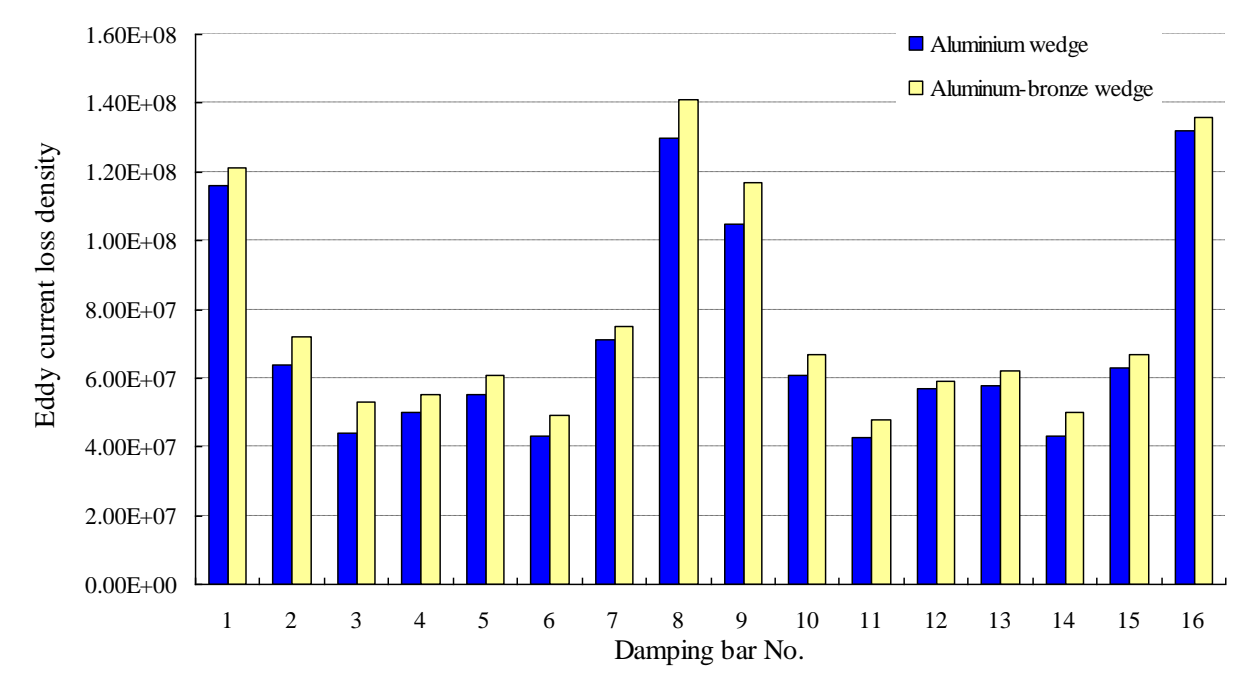

Figure 7. The Loss Density Distribution of Damping Bars

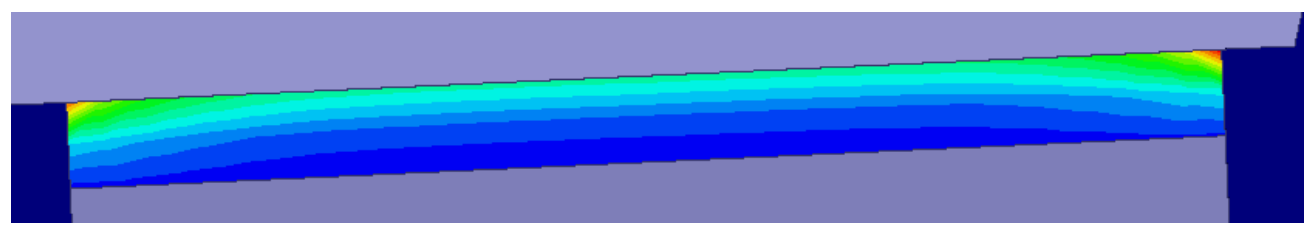

Figure 8. The Loss Density Distribution of Each Damping Bar

It can be seen from Figure 7 and 8 that under the same wedge material condition, the eddy current loss density of damping winding is much higher than the wedge's, that is also the reason why the damping bar is the most easily damaged part of half-speed large generator. The eddy current loss density of the interchange where the rotor teeth, slot wedges and damping bars meet, is the maximum, and it is also the most easily burnt position.

\section{Conclusions}

The negative-sequence eddy current distribution of AP1000 generator rotor surface is deeply studied in this work. Meanwhile, the slot wedge materials' different influences on the rotor surface loss density are also obtained. By using the finite element analysis method, the two dimensional mathematical model of AP1000 generator is got. Then, the rotor surface's eddy current loss density variety rules of two kinds of material, aluminium and aluminium bronze, are calculated and obtained.

From the analysis results, it can be seen clearly that slot wedge loss density decreases gradually from the upper part to the lower part, eddy current loss density of the big tooth area is large, and the big teeth are more easily overheat and burned than the small teeth part of the rotor. Moreover, damping bars have the biggest loss 
density of the rotor surface, and there is a close relationship between the eddy current density and the slot wedge material.

\section{Acknowledgements}

This work was supported by the National Major Scientific and Technological Special Project of China (No.2009ZX06004-013-04-01, Sub-project No.2012BAF03B01-X).The authors appreciate the financial support from the National Major Scientific and Technological Special Project of the Chinese Government. And Harbin Electric Machinery Company Limited, Harbin, China, is also acknowledged for the technical support for this work.

\section{References}

[1] T. Bach, D. Wohner and K. Takahashi, "Determining Negative Sequence Currents of Turbine Generator Rotors", Electrical Machines and Systems, vol. 1, no. 6, (2009).

[2] L. Jinxiang, S. Yutian and Y. Guijie, "Calculation and analysis of 3D magnetic field for end region of large turbo generators", Electrical Machines and Systems, vol. 3, no. 2079, (2005).

[3] B.-J. Ge, W. Guo and D.-H. Zhang, "Harmonic Analysis of AP1000 Large-capacity Turbo-generator Based on BP Neural Network", International Journal of Control and Automation, vol. 6, no. 163, (2013).

[4] M. Fujita, T. Yoda and H. Tsuda, "Magnetic field analysis of stator core end region of large turbogenerators", Magnetics, vol. 36, no. 1850, (2000).

[5] H. Liangliang, S. Yuguang and Q. Ami, "Analysis on the Negative Sequence Impedance Directional Protection for Stator Internal Fault of Turbo Generator", Electrical Machines and Systems, (2010), pp. 1421-1424.

[6] D. Jianyang, L. Xubiao, L. Feng and Y. Shiyou, "Negative Sequence Eddy Current Field Analysis of a 1055 MVA Turbo-generator by using 3D Finite Element Methods", ICEMS, vol. 3, no. 2075, (2005).

[7] J. Hui-yong, L. Wei-li, D. Shu-ye and X. Bin, "Analysis of 3D Fluid Fields in Large Synchronous Generator Stator", ICSC, (2005), pp. 1242-1246.

[8] G. Bao-jun, A. Wang-qiang, T. Da-jun and L. Peng, "Simulation of the Temperature Field of Heliumcolled Driving Motor Rotor End", Journal of Harbin University of Science and Technology, vol. 19, no. 4, (2014).

[9] IEEE Std.1459-2000, IEEE trial-use standard definitions for the measurement of electric power quantities under sinusoidal and non-sinusoidal balanced condition, New York, USA, (2000).

[10] N.-Q. Dinh and J. Arrillaga, "A Salient-pole Generator Model for Harmonic Analysis", IEEE Transactions on power systems, vol. 4, no. 609, (2001).

[11] K. Richter Sedlazeck and C. Strack, “Type Testing a 2000 MW Turbogenerator”, Electric Machines and Drives Conference, (2009), pp. 465-470.

[12] M. Fujita, T. Yoda, H. Tsuda and H. Ito, "Magnetic field analysis of stator core end region of large turbogenerators", Magnetics, vol. 36, no. 1850, (2000).

[13] L. Jinxiang, S. Yutian and Y. Guijie, "Calculation and analysis of 3D magnetic field for end region of large turbo generators", Electrical Machines and Systems, vol. 3, no. 2079, (2005).

[14] B. Irwanto, K. Steigleder and O. Perros, "Large $60 \mathrm{~Hz}$ turbogenerators Mechanical design and improvements", Electric Machines and Drives Conference, (2009), pp. 471-476.

[15] W. Yixuan, W. Ying and Q. Haifei, "Dynamic Design and Simulation Analysis of 1000MW Large Turbo-Generator", Mechatronics and Automation, (2009), pp. 1650-1655.

[16] M. G. Pantelyat and N. G. Shulzhenko, "Finite element analysis of electromagnetic field and losses in a turbogenerator rotor", Computational Electromagnetics, (2006). 
International Journal of Control and Automation Vol. 8, No. 6 (2015) 